\title{
Orange juice combined to a healthy-eating pattern improved endothelial function and reduced global risk of CHD in metabolic syndrome patients
}

\section{Abstract}

Current evidence has shown that orange juice flavonoids have antihypertensive, lipid lowering, insulin sensitizing, antioxidant and antinflammatory properties, which are correlated with antiatherogenic activity. The objective of this study was to verify whether regular administration of $100 \%$ pure orange juice $(\mathrm{OJ})$, as a source of antioxidant and bioactive compounds, associated with a healthy dietary pattern improves endothelial and vascular function and reduces Global Risk of Coronary Heart Disease (Global Risk of CHD). Obese volunteers $(n=68)$ with metabolic syndrome were randomly assigned to the control $(n=34)$ or OJ $(n=34)$ groups, and all of them were counseled and followed up to maintain a healthy-eating pattern for 12 weeks. In addition, OJ group participants drank $500 \mathrm{ml}$ of $100 \%$ orange juice daily in two servings of $250 \mathrm{~mL}$ each between meals. After the intervention, a significant reduction of $2 \%$ in anthropometric measurements (body weight and fat) was observed in both groups, with no change in lean mass. In the OJ group, a significant reduction of total cholesterol $(-9.4 \%)$ was detected, whereas the controls reduced only HDL-C (-9\%). In both groups there was a significant reduction in systolic and diastolic blood pressure $(-8 \%$ and $-9 \%$, respectively) and an increase in antioxidant capacity $(1 \%)$. Only the OJ group reduced vascular markers ICAM (-14\%) and VCAM (-15\%) and inflammatory markers TNF-alpha $(-20 \%)$ and hsPCR $(-22 \%)(\mathrm{p}<0.05)$. Both groups reduced IL-6 $(-45 \%)$, improved brachial artery flow-mediated dilatation (BA-FMD), and reduced carotid artery intima-media thickness $($ CA-IMT) by $10 \%(\mathrm{p}<0.05)$. Daily consumption of orange juice, along with a healthy-eating pattern, was able to reduce cardiovascular risk factors related to systemic inflammation and endothelial function in individuals with metabolic syndrome. A significant reduction in the prevalence of the high-risk of CHD was observed in $47 \%$ of subjects who consumed a healthy diet, while $70 \%$ of patients who had a healthy eating pattern along with orange juice reverted to low CHD risk, showing the additional benefit of regular intake of $100 \%$ orange juice. This was probably due to the action of antioxidant compounds present in high concentrations in orange juice, such as vitamin $\mathrm{C}$ and citrus flavonoids

\section{Conflict of Interest}

There is no conflict of interest 